\title{
NEST GENERATED INTERSECTION RINGS IN TYCHONOFF SPACES
}

\author{
BY \\ A. K. STEINER AND E. F. STEINER
}

Tychonoff spaces were characterized by Frink [4] as $T_{1}$-spaces possessing normal bases. The zero-sets of continuous real-valued functions provide the best known normal base, but in general there are many others. They need not consist entirely of zero-sets. Each normal base leads to a Hausdorff compactification (called Wallman because of the construction used). If the normal base consists entirely of zerosets, the compactification has been called a $Z$-compactification [15]. This generalization of the Wallman procedure was also considered earlier by Sanin [12] and Banaschewski [2], but apparently Frink was first to consider whether all Hausdorff compactifications might be obtained in this way.

Frink was also concerned about the hereditary property of complete regularity and asked for a direct internal proof of this using the normal base characterization. A complication lies in the fact that the trace of a normal base on a subspace need not be a normal base. In [13], one of the authors showed that completely regular spaces (not necessarily $T_{1}$ ) are characterized by possessing a stronger base, the trace of which forms a base with the same properties. These bases are called separating nest generated intersection rings. The hereditary property of such rings shows the hereditary property of complete regularity.

In this paper we consider the Wallman compactification $\omega(X, \mathscr{F})$ and the Wallman realcompactification $v(X, \mathscr{F})$ associated with a given separating nest generated intersection ring. $v(X, \mathscr{F})$ is constructed in $\omega(X, \mathscr{F})$ in the same way that the Hewitt realcompactification $v(X)$ is constructed in the Stone-Čech compactification $\beta(X)$.

Alo and Shapiro [1] have considered normal bases which are intersection rings (but not necessarily nest generated). Following the analogy with $v(X)$ in $\beta(X)$, they construct a space $\eta(X)$ and show that in many cases it is realcompact. They ask whether this is always the case and also whether all realcompactifications may be obtained in this manner. In $\S 3$ we provide examples which give negative answers to both of these questions. Thus nest generation cannot be relaxed and still keep $v(X, \mathscr{F})$ realcompact.

In $\$ 4$ we investigate the relation between separating nest generated intersection rings and certain inverse-closed subalgebras of $C(X)$ (see [6], [7], [9]). Earlier in

Presented to the Society, August 27, 1969; received by the editors June 23, 1969.

Copyright (C) 1970, American Mathematical Society 
$\S 2$ we show that for such a $\operatorname{ring} \mathscr{F}, \omega(X, \mathscr{F})$ is a $Z$-compactification, in fact $\mathscr{F}$ consists of the traces on $X$ of all zero-sets in $\omega(X, \mathscr{F})$. This is not the case for all normal bases. Hager [6], has shown that the zero-sets of certain inverse-closed subalgebras of $C(X)$ (called algebras) also have this property. Indeed, we show there is a one-to-one correspondence between algebras and separating nest generated intersection rings. Thus theorems about $\omega(\mathscr{F})$ and $v(\mathscr{F})$ are equivalent to theorems concerning certain spaces of maximal ideals. Although we will give direct proofs here, several have been proved in [6], [7], and [9]. We will indicate the sources of these earlier proofs.

In $\S 5$ we consider the family $\mathscr{L}^{*}(X)$ of all compactifications provided by separating nest generated intersection rings on $X$. In some cases $\mathscr{L}^{*}(X)$ has only one element, but it can be arbitrarily large. If $X$ is locally compact, $\mathscr{L}^{*}(X)$ is a complete lattice under the usual ordering of compactifications, but is not always a sublattice of the lattice of all Hausdorff compactifications. Thus the algebras in $C(X)$ also form a complete lattice.

\section{Preliminaries.}

1.1 A family $\mathscr{F}$ of closed subsets of $X$ will be called separating if for each closed set $H \subset X$ and each point $p, p \notin H$, there are disjoint sets in $\mathscr{F}$, one containing $H$ and the other containing $p$. The family $\mathscr{F}$ will be called a ring if it is closed under finite unions and finite intersections, and will be called an intersection ring if it a ring which is closed under countable intersections. A sequence $\left\{F_{n}\right\}$ of sets in $\mathscr{F}$ is called a nest in $\mathscr{F}$ if there is a sequence $\left\{H_{n}\right\}$ in $\mathscr{F}$ such that $X-H_{n+1} \subset F_{n+1}$ $\subset X-H_{n} \subset F_{n}$ for $n=1,2, \ldots$ A family $\mathscr{F}$ of closed sets is nest generated if for each member $F$ of $\mathscr{F}$ there is a nest $\left\{F_{n}\right\}$ in $\mathscr{F}$ such that $F=\bigcap\left\{F_{n}: n \in N\right\}$. Throughout the rest of the paper we will let $\mathscr{L}(X)$ denote the family of all separating, nest-generated intersection rings on $X$.

1.2 A family $\mathscr{F}$ of closed subsets of $X$ is called normal if disjoint sets in $\mathscr{F}$ can be separated by disjoint complements of sets in $\mathscr{F}$. Frink [4] showed that a $T_{1}$-space is completely regular if and only if it has a separating, normal ring. In [13] the second author introduced the concept of a separating nest generated intersection ring which is stronger than that of a separating normal ring and has the additional property that the trace of a separating nest generated intersection ring on any subspace is also such a ring.

1.3 Lemma. If $\mathscr{F} \in \mathscr{L}(X)$ then $\mathscr{F}$ is normal.

(Proof given in [13].)

1.4 Lemma. If $\mathscr{F} \in \mathscr{L}(X)$ and $Y \subset X$ then $\{F \cap Y: F \in \mathscr{F}\} \in \mathscr{L}(Y)$.

(Proof given in [13].)

We now state and outline the proof of some facts concerning nest generated families which will be needed. 
1.5 LEMma. If $\mathscr{F}$ is a nest generated family then the ring $\mathscr{F}_{1}$ of finite intersections of finite unions of members of $\mathscr{F}$ is also nest generated.

Proof. Let $K=\bigcup\left\{G_{i} \in \mathscr{F}: 1 \leqq i \leqq m\right\}$. For each $i, 1 \leqq i \leqq m, G_{i}=\bigcap\left\{F_{i, n}: n \in N\right\}$ where $\left\{F_{i, n}\right\}$ is a nest in $\mathscr{F}$. If $A_{n}=\bigcup\left\{F_{i, n}: 1 \leqq i \leqq m\right\}$ then $\left\{A_{n}\right\}$ is a nest in $\mathscr{F}_{1}$ and $K=\bigcap\left\{A_{n}: n \in N\right\}$.

Let $L=\bigcap\left\{K_{i}: 1 \leqq i \leqq m\right\}$ where each $K_{i}$ is a finite union of members of $\mathscr{F}$. For each $i, 1 \leqq i \leqq m, K_{i}$ is the intersection over a nest $\left\{A_{i, n}\right\}$ in $\mathscr{F}_{1}$ and if $C_{n}=$ $\bigcap\left\{A_{i, n}: 1 \leqq i \leqq m\right\}$ then $\left\{C_{n}\right\}$ is a nest in $\mathscr{F}_{1}$ and $L=\bigcap\left\{C_{n}: n \in N\right\}$.

1.6 LEMMA. If $\mathscr{F}$ is a nest generated ring and $\mathscr{F}_{1}$ is the family of countable intersections of members of $\mathscr{F}$, then $\mathscr{F}_{1}$ is nest generated.

Proof. Let $K=\bigcap\left\{G_{i} \in \mathscr{F}: i \in N\right\}$ where for each $i, G_{i}$ is the intersection of the nest $\left\{F_{i, n}\right\}$ in $\mathscr{F}$. If for each $n \in N, A_{n}=\bigcap\left\{F_{i, n}: 1 \leqq i \leqq n\right\}$, then $\left\{A_{n}\right\}$ is a nest in $\mathscr{F}$ and $K=\bigcap\left\{A_{n}: n \in N\right\}$. Thus $\mathscr{F}_{1}$ is nest generated.

1.7 LEMMA. If $\mathscr{F}$ and $\mathscr{G}$ are nest generated intersection rings, then the ring $\mathscr{F}+\mathscr{G}$ of countable intersections of finite unions of members of $\mathscr{F} \cup \mathscr{G}$ is a nest generated intersection ring. Moreover if $\mathscr{F}$ or $\mathscr{G}$ is separating, then so is $\mathscr{F}+\mathscr{G}$.

Proof. Clearly $\mathscr{F}+\mathscr{G}$ is an intersection ring and since $\mathscr{F} \cup \mathscr{G}$ is nest generated, by Lemmas 1.5 and 1.6 , so is $\mathscr{F}+\mathscr{G}$.

1.8 If $\mathscr{F}$ is a separating normal ring on $X$ then the Wallman space, $\omega(X, \mathscr{F})$, of all ultrafilters on $\mathscr{F}$ is a Hausdorff compactification of $X$, [2], [4], [12], [14]. When there is no question as to the space $X$, we will simply write $\omega(\mathscr{F})$.

1.9 Using the notation of [5], $C(X)$ will denote the ring of all continuous realvalued functions on $X, C^{*}(X)$ will denote the bounded functions in $C(X)$ and $Z[X]$ will denote the family of zero-sets on $X, Z[X]=\left\{f^{\leftarrow}(0): f \in C(X)\right\}$. A cozeroset is the complement of a zero-set. All spaces will be assumed to be Tychonoff.

\section{Wallman compactifications.}

2.1 If $\mathscr{F} \in \mathscr{L}(X)$ then by Lemma $1.3, \mathscr{F}$ is a normal separating ring; so, $\omega(\mathscr{F})$ is a Hausdorff compactification. It is known [5] that every zero-set in $\beta X$ is a countable intersection of closures of zero-sets in $X$. If $\mathscr{F} \in \mathscr{L}(X)$ then $\omega(\mathscr{F})$ satisfies the analogous condition: every zero-set in $\omega(\mathscr{F})$ is a countable intersection of closures of sets in $\mathscr{F}$. The intersection of the zero-sets in $\beta X$ with $X$ gives the zero-sets in $X$; the intersections of the zero-sets in $\omega(\mathscr{F})$ with $X$ give $\mathscr{F}$.

2.2 Theorem. If $\mathscr{F} \in \mathscr{L}(X)$ then $\mathscr{F}$ is precisely the trace on $X$ of all zero-sets in the Wallman compactification, $\omega(X, \mathscr{F})$.

Proof. Let $F \in \mathscr{F} . \mathscr{F}$ is nest generated so $F=\bigcap\left\{X-H_{n}: n \in N\right\}$ where for each $n$, $H_{n} \in \mathscr{F}$. From the definition of $\omega(\mathscr{F})$, the closures of $F$ and $H_{n}$ in $\omega(\mathscr{F})$ are disjoint, so there exist zero-sets $Z_{n} \in Z[\omega(\mathscr{F})]$ such that $\operatorname{cl} F \subset Z_{n} \subset \omega(\mathscr{F})-\mathrm{cl} H_{n}$. Thus $\operatorname{cl} F \subseteq Z=\bigcap\left\{Z_{n}: n \in N\right\} \subseteq \bigcap\left\{\omega(\mathscr{F})-\operatorname{cl} H_{n}: n \in N\right\}$ and so

$$
F \subseteq Z \cap X \subseteq \bigcap\left\{X-H_{n}: n \in N\right\}
$$


Conversely, if $Z \in Z[\omega(\mathscr{F})]$ then $Z$ is the intersection of a countable number of open subsets $\left\{U_{n}\right\}$ in $\omega(\mathscr{F})$. Since the family of closures of members of $\mathscr{F}$ forms a base for the closed sets in $\omega(\mathscr{F})$, for each $n \in N$ there is an $F_{n} \in \mathscr{F}$ such that $Z \subset \operatorname{cl} F_{n} \subset U_{n}$. Thus $Z=\bigcap\left\{\operatorname{cl} F_{n}: n \in N\right\}$ and intersecting with $X$ gives

$$
Z \cap X=\bigcap\left\{F_{n}: n \in N\right\} \in \mathscr{F} \text {. }
$$

2.3 CoRollary. If $\mathscr{F}, \mathscr{G} \in \mathscr{L}(X)$ then $\mathscr{F}=\mathscr{G}$ if and only if $\omega(\mathscr{F})=\omega(\mathscr{G})$.

2.4 We note that the relation stated in Corollary 2.3 does not hold for normal bases. For example, let $X$ be the discrete space of cardinality $c, \mathscr{F}=\{F \subset X: F$ finite or $X-F$ finite $\}$ and $\mathscr{G}=\{F \subset X: F$ finite or $X-F$ countable $\} . \mathscr{G}$ is in $\mathscr{L}(X)$ but $\mathscr{F}$ is not. $\omega(\mathscr{F})=\omega(\mathscr{G})$ is the one point compactification of $X$.

2.5 We will define $\mathscr{L}^{*}(X)$ to be the family of all Hausdorff compactifications of $X$ of the form $\omega(\mathscr{F})$, for some $\mathscr{F} \in \mathscr{L}(X)$. The family $Z[X]$ of all zero-sets on $X$ is in $\mathscr{L}(X)$ so the Stone-Čech compactification of $X, \beta X$, is in $\mathscr{L}^{*}(X)$ since $\beta X=\omega(Z[X])$.

2.6 THEOREM. The one-point compactification $X^{*}$ of a noncompact locally compact space $X$ is in $\mathscr{L}^{*}(X)$ if and only if $X$ is not Lindelöf.

Before proving this theorem we need the following lemma.

2.7 LEMmA. If $X$ is a Lindelöf space then $\mathscr{L}(X)=\{Z[X]\}$.

Proof. By Theorem 2.2, if $\mathscr{F} \in \mathscr{L}(X)$ then $\mathscr{F}$ is the trace on $X$ of all zero-sets in $\omega(X, \mathscr{F})$. But by 5.3 of [8], due to M. Jerison, the trace on $X$ of all zero-sets in $\omega(X, \mathscr{F})$ is just $Z[X]$. Thus $\mathscr{L}(X)=\{Z[X]\}$.

Proof of theorem. If $X$ is Lindelöf, $\mathscr{L}(X)=\{Z[X]\}$ so $\mathscr{L}^{*}(X)=\{\beta X\}$. Since $X$ is Lindelöf and not compact, $X$ is realcompact and not pseudocompact. Thus $|\beta X-X| \neq 1[5]$ and $\beta X \neq X^{*}$.

Conversely, assume $X$ is not Lindelöf. If $\{p\}=X^{*}-X$, then the complement of any open set containing $p$ is compact. If $\{p\}$ were a $G_{\delta}$ then $X$ would be $\sigma$-compact, and thus Lindelöf, so $\{p\}$ is not a zero-set. The family $Z\left[X^{*}\right]$ is a base for the closed sets in $X^{*}$ and has the trace property with respect to $X$ (i.e. if $Z_{1}, Z_{2} \in Z\left[X^{*}\right]$ and $Z_{1} \cap Z_{2} \cap X=\varnothing$, then $\left.Z_{1} \cap Z_{2}=\varnothing\right)$. Thus by Theorem 2 of [14], if $\mathscr{F}$ is the trace on $X$ of zero-sets in $X^{*}$, then $X^{*}=\omega(\mathscr{F})$. By Lemma $1.4 \mathscr{F} \in \mathscr{L}(X)$. Thus $X^{*} \in \mathscr{L}^{*}(X)$.

Every family in $\mathscr{L}(X)$ gives a Hausdorff compactification of $X$, but not every compactification $Y$ of $X$ can be obtained in this way, although the trace on $X$ of the zero-sets in $Y$ is in $\mathscr{L}(X)$. The following order does hold, however.

2.8 LEMMA. If $Y$ is a Hausdorff compactification of $X$ and $\mathscr{F}=\{Z \cap X: Z \in Z[Y]\}$ then $\omega(\mathscr{F}) \geqq Y$ (Hager $[6, p .244])$.

Proof. To show that $\omega(\mathscr{F}) \geqq Y$ we will show that the identity map on $X$ has a continuous extension from $\omega(\mathscr{F})$ onto $Y$. We will denote the closure of $F$ in $\omega(\mathscr{F})$ by $\mathrm{cl} F$ and in $Y$ by $F^{-}$. 
For $p \in \omega(\mathscr{F})$ define $\varphi(p)=\bigcap\left\{F^{-}: F \in \mathscr{F}\right.$ and $\left.p \in \operatorname{cl} F\right\}$. The family $\{F \in \mathscr{F}:$ $p \in \operatorname{cl} F\}$ has the finite intersection property (from the definition of $\omega(\mathscr{F})$ ) so $\bigcap\left\{F^{-}: F \in \mathscr{F}\right.$ and $\left.p \in \mathrm{cl} F\right\} \neq \varnothing$. Distinct points in $Y$ can be separated by disjoint zero-set neighborhoods, so $\varphi(p)$ is precisely one point.

If $p \in X$ then $p \in \operatorname{cl} F$ if and only if $p \in F$ so $\varphi(p)=p$. If $y \in Y$ then $y$ is the intersection of all its zero-set neighborhoods, so if $p \in \bigcap\{\operatorname{cl}(Z \cap X): Z$ is a zero-set neighborhood of $y$ in $Y\}$, then $\varphi(p)=y$.

For each closed set $H$ in $Y, \varphi^{-}[H]=\bigcap\{\operatorname{cl}(Z \cap X): Z$ is a zero-set neighborhood of $H$ in $Y$ \} and thus $\varphi$ is continuous.

This lemma allows us to prove an analogue to the theorem in [5] that if $X \subset T$ $\subset \beta X$ then $\beta T=\beta X$.

2.9 TheOREM. If $\mathscr{F} \in \mathscr{L}(X), X \subset T \subset \omega(X, \mathscr{F})$ and

$$
\mathscr{F}^{\prime}=\{Z \cap T: Z \in Z[\omega(X, \mathscr{F})]\},
$$

then $\omega\left(T, \mathscr{F}^{\prime}\right)=\omega(X, \mathscr{F})$.

Proof. By Lemma $2.8, \omega\left(T, \mathscr{F}^{\prime}\right) \geqq \omega(X, \mathscr{F}) . X$ is dense in $T$ so $\omega\left(T, \mathscr{F}^{\prime}\right)$ is a Hausdorff compactification of $X$ and if $\mathscr{G}=\left\{Z \cap X: Z \in Z\left[\omega\left(T, \mathscr{F}^{\prime}\right)\right]\right\}$, by Lemma 2.8 again, $\omega(X, \mathscr{G}) \geqq \omega\left(T, \mathscr{F}^{\prime}\right)$. But if $Z \in Z\left[\omega\left(T, \mathscr{F}^{\prime}\right)\right]$ then $Z \cap T \in \mathscr{F}^{\prime}$ so $Z \cap T$ $=Z^{\prime} \cap T$ for some $Z^{\prime} \in Z[\omega(X, \mathscr{F})]$. Thus $Z \cap X=Z^{\prime} \cap X \in \mathscr{F}$ and $\mathscr{G} \subset \mathscr{F}$. From [15] it follows that $\omega(X, \mathscr{F}) \geqq \omega(X, \mathscr{G})$ and the desired equality results.

2.10 Corollary. If $\mathscr{F} \in \mathscr{L}(X)$ and $T$ is a cozero-set of $\omega(X, \mathscr{F})$ containing $X$, then $\beta T=\omega(X, \mathscr{F})$ (Isbell $[9,1.17])$.

Proof. If $T=\omega(X, \mathscr{F})-Z(f)$ for some $f \in C(\omega(X, \mathscr{F}))$ and $g=|f| \wedge 1$, then $T=\bigcup\left\{g^{\leftarrow}([1 / n, 1]): n=2,3, \ldots\right\} . T$ is thus $\sigma$-compact, hence Lindelöf, so $\mathscr{L}(T)=\{Z[T]\}$. If $\mathscr{F}^{\prime}=\{Z \cap T: Z \in Z[\omega(X, \mathscr{F})]\}$ then $\mathscr{F}^{\prime}=Z[T]$, so $\beta T=$ $\omega\left(T, \mathscr{F}^{\prime}\right)=\omega(X, \mathscr{F})$.

\section{Wallman realcompactifications.}

3.1 Let $\mathscr{F} \in \mathscr{L}(X)$ and define $v(X, \mathscr{F})$ to be the family of all ultrafilters on $\mathscr{F}$ which have the countable intersection property. $X \subseteq v(X, \mathscr{F}) \subseteq \omega(X, \mathscr{F})$ and the topology on $v(X, \mathscr{F})$, taking sets of the form $F^{*}=\{q \in v(X, \mathscr{F}): F \in q\}$, for $F \in \mathscr{F}$, as a base for the closed sets, coincides with the topology on $v(X, \mathscr{F})$ considered as a subspace of $\omega(X, \mathscr{F})$. When there is no question as to the space $X$, we will write $v(X, \mathscr{F})$ as simply $v(\mathscr{F})$.

3.2 TheOREM. For $\mathscr{F} \in \mathscr{L}(X), v(\mathscr{F})$ is a realcompact space $($ Isbell $[9, p .117]$ and Hager [6, 5.3(c)]).

Proof. First we show that for every zero-set $Z \in Z[\omega(\mathscr{F})], Z \cap v(\mathscr{F}) \neq \varnothing$ implies $Z \cap X \neq \varnothing$. Let $q \in Z \cap v(\mathscr{F})$. $Z$ is a countable intersection of zero-set neighborhoods $\left\{Z_{n}\right\}$, and $Z \subset \operatorname{cl}\left(Z_{n} \cap X\right) \subset Z_{n}$ for each $n$. Thus $q \in \operatorname{cl}\left(Z_{n} \cap X\right)$ so $Z_{n} \cap X$ is in the ultrafilter $q$. This ultrafilter has the countable intersection property so $Z \cap X=\bigcap\left\{Z_{n} \cap X: n \in N\right\} \neq \varnothing$. 
Now, for each point $p \in \omega(\mathscr{F})-v(\mathscr{F})$, we show there is a zero-set containing $p$, missing $v(\mathscr{F}) . p$ is an ultrafilter on $\mathscr{F}$ which fails to have the countable intersection property. Thus there are sets $F_{n} \in p$ such that $\bigcap\left\{F_{n}: n \in N\right\}=\varnothing$. By Theorem 2.2, $F_{n}=Z_{n} \cap X$ for some $Z_{n} \in Z[\omega(\mathscr{F})]$, so if $Z=\cap\left\{Z_{n}: n \in N\right\}$ then $Z \cap X=\varnothing$. Since $p \in \operatorname{cl} F_{n} \subset Z_{n}$ for each $n, p \in Z$, and since $Z \cap X=\varnothing, Z \cap v(\mathscr{F})=\varnothing$.

Each cozero-set in a compact space is realcompact [5] and $v(\mathscr{F})$ is the intersection of all the cozero-sets containing it and, as such, is realcompact.

3.3 As a result of Theorem 3.2, a realcompact space will be called a Wallman realcompactification of $X$ if it is of the form $v(X, \mathscr{F})$ for some $\mathscr{F} \in \mathscr{L}(X)$. The Hewitt realcompactification $v X$ is just $v(Z[X])$.

Notice that $v(\mathscr{F})$ need not be $X$ even when $X$ is realcompact. In the example in 2.4, $v(X, \mathscr{G})=\omega(X, \mathscr{G}) \neq X$ and $X$ is realcompact.

In fact, as $\omega(X, \mathscr{F})$ gets smaller, $v(X, \mathscr{F})$ becomes "larger" in the following sense.

3.4 THEOREM. If $\omega\left(\mathscr{F}_{1}\right) \geqq \omega\left(\mathscr{F}_{2}\right)$ and $\varphi$ is the canonical quotient map from $\omega\left(\mathscr{F}_{1}\right)$ onto $\omega\left(\mathscr{F}_{2}\right)$ then $\varphi\left(v\left(\mathscr{F}_{1}\right)\right) \subseteq v\left(\mathscr{F}_{2}\right)$.

Proof. If $p \in \varphi\left(v\left(\mathscr{F}_{1}\right)\right)-v\left(\mathscr{F}_{2}\right)$ then there is a zero-set $Z$ in $Z\left[\omega\left(\mathscr{F}_{2}\right)\right]$ such that $p \in Z$ and $Z \cap v\left(\mathscr{F}_{2}\right)=\varnothing$ (Theorem 3.1). Thus $Z \cap X=\varnothing$ and $\varphi^{\leftarrow}[Z]$ is a zeroset in $Z\left[\omega\left(\mathscr{F}_{1}\right)\right]$ which meets $v\left(\mathscr{F}_{1}\right)$ but misses $X$. This contradiction proves that $\varphi\left(v\left(\mathscr{F}_{1}\right)\right) \subseteq v\left(\mathscr{F}_{2}\right)$.

Not all realcompactifications of a space $X$ can be obtained as $v(X, \mathscr{F})$ for some $\mathscr{F} \in \mathscr{L}(X)$. In fact, in some cases, only the Hewitt realcompactification can be obtained this way.

3.5 Lemma. If $X$ is realcompact but not Lindelöf then there are at least two Wallman realcompactifications of $X$.

Proof. If $X$ is not Lindelöf then there is a filter $\mathscr{A}$ of zero-sets in $Z[X]$ which is closed under countable intersections but $\cap \mathscr{A}=\varnothing$. Let $\mathscr{F}=\{Z \in Z[X]: Z \in \mathscr{A}$ or $Z \cap A=\varnothing$ for some $A \in \mathscr{A}\}$. It is clear that $\mathscr{F}$ is an intersection ring. If $H$ is a closed subset of $X$ and $p \notin H$, then there is a set $A \in \mathscr{A}$ such that $p \notin A$. There are disjoint zero-sets $Z_{1}$ and $Z_{2}$ such that $p \in Z_{1}$ and $H \cup A \subset Z_{2}$. Thus $Z_{2} \in \mathscr{A}$ and $Z_{1} \cap Z_{2}=\varnothing$ so $Z_{1}, Z_{2} \in \mathscr{F}$ and $\mathscr{F}$ is separating. Now we will show that $\mathscr{F}$ is nest generated. Every zero-set is the intersection of a nest of zero-sets and if $Z \in \mathscr{A}$ then clearly the nest for $Z$ is in $\mathscr{F}$. If $Z \in \mathscr{F}$ and $Z \cap A=\varnothing$ for $A \in \mathscr{A}$ then $Z=\bigcap\left\{X-Z_{i}: i \in N\right\}$. Inductively choose zero-sets $F_{i}, E_{i}$ such that $Z \subset X-E_{1}$ $\subset F_{1} \subset X-\left(Z_{1} \cup A\right)$ and $Z \subset X-E_{i} \subset F_{i} \subset X-\left(Z_{i-1} \cup E_{i-1}\right)$ for $i>1$. For each $i, E_{i} \in \mathscr{A}$ and $F_{i} \cap A=\varnothing$, so $\left\{F_{n}\right\}$ is a nest in $\mathscr{F}$. Obviously $Z=\bigcap\left\{F_{n}: n \in N\right\}$. Thus $\mathscr{F} \in \mathscr{L}(X)$. Now $\mathscr{A}$ is a free ultrafilter on $\mathscr{F}$ with the countable intersection property, so $\mathscr{A} \in v(\mathscr{F})$. Thus $X=v X$ and $v(\mathscr{F})$ are two distinct Wallman realcompactifications of $X$. 
3.6 LEMMA. If $X$ is not realcompact and not almost compact then there are at least two Wallman realcompactifications of $X$.

Proof. The hypothesis implies that $|\beta X-X|>1$ and $|v X-X| \geqq 1$. Let $q \in v X-X$, $p \in \beta X-X, p \neq q$, and let $Z_{p}$ and $Z_{q}$ be disjoint zero-set neighborhoods of $p$ and $q$ in $\beta X$. If $Y$ is the compactification of $X$ obtained by identifying $p$ and $q$ then $Z_{p} \cap X$ is not the trace of a zero-set in $Y$ on $X$. For, if there were a zero-set $Z \in Z[Y]$, such that $Z \cap X=Z_{p} \cap X$ then $\varphi^{\leftarrow}[Z]$, where $\varphi$ is the canonical quotient map from $\beta X$ onto $Y$, would be zero-set in $\beta X$ and $\varphi^{\leftarrow}[Z] \cap Z_{q}$ would be a zero-set containing $q \in v X$ but missing $X$. Thus $\mathscr{F}=\{Z \cap X: Z \in Z[Y]\} \in \mathscr{L}(X)$ and $\mathscr{F} \neq Z[X]$. Since $Z_{p} \cap X$ and $Z_{q} \cap X$ have disjoint closures in $v X$ but not in $v(\mathscr{F}), v(\mathscr{F}) \neq v X$.

3.7 THEOREM. A space $X$ has precisely one Wallman realcompactification if and only if it is Lindelöf or almost compact (Hager and Johnson [7, Theorem 3]).

Proof. If $X$ is almost compact then $X$ has only one Hausdorff compactification, $\beta X$, and thus only one realcompactification, $v X=\beta X$. If $X$ is Lindelöf then by Lemma $2.7, \mathscr{L}(X)=\{Z[X]\}$ so the only Wallman realcompactification is $v X$. Lemmas 3.5 and 3.6 prove the converse.

3.8 COROLlary. If $X$ is not Lindelöf and not pseudocompact then $X$ has infinitely many Wallman realcompactifications.

Proof. If $X$ is not realcompact then $|v X-X| \geqq 1$ and since $X$ is not pseudocompact, $|\beta X-v X| \geqq 2^{c}$. There are an infinite number of choices for the point $p$ of Lemma 3.6 and each choice gives a different Wallman realcompactification.

If $X$ is realcompact, the proof of Lemma 3.5 needs to be extended by induction. Let $\mathscr{A}_{1}=\mathscr{A}$ and $\mathscr{F}_{1}=\mathscr{F}$ be as in 3.5. Assume that for each positive integer $k<n$, $\mathscr{A}_{k}$ and $\mathscr{F}_{k}$ have been defined so that $\mathscr{A}_{k}$ is a filter of zero-sets, closed under countable intersections and $\cap \mathscr{A}_{k}=\varnothing ; \mathscr{F}_{k}=\left\{Z \in Z[X]: Z \in \mathscr{A}_{k}\right.$ or $Z \cap A=\varnothing$ for some $\left.A \in \mathscr{A}_{k}\right\} ; \mathscr{A}_{k-1} \varsubsetneqq \mathscr{A}_{k}$; and the associated Wallman realcompactifications $v\left(\mathscr{F}_{k}\right)$ are all distinct.

Since $X$ is realcompact and $\mathscr{A}_{n-1}$ has the countable intersection property with $\cap \mathscr{A}_{n-1}=\varnothing, \mathscr{A}_{n-1}$ does not converge in $\beta X$ and thus can be extended to two distinct ultrafilters of zero-sets, $\mathscr{U}$ and $\mathscr{V}$ [5]. There are disjoint zero-sets $Z \in \mathscr{U}$ and $Z^{\prime} \in \mathscr{V}$. Letting $\mathscr{A}_{n}$ be the filter generated by $\mathscr{A}_{n-1}$ and $Z$, we see that $\mathscr{A}_{n}$ is closed under countable intersections, $\bigcap \mathscr{A}_{n}=\varnothing$, and $\mathscr{A}_{n-1} \varsubsetneqq \mathscr{A}_{n}$. If $\mathscr{F}_{n}$ is defined as above, then $\mathscr{F}_{n} \in \mathscr{L}(X)$. Since $Z^{\prime}$ is closed in $v\left(\mathscr{F}_{n}\right)$ but not in $v\left(\mathscr{F}_{k}\right)$ for $k<n$, $v\left(\mathscr{F}_{n}\right) \neq v\left(\mathscr{F}_{k}\right)$ for all $k<n$.

3.9 If $\mathscr{F} \in \mathscr{L}(X)$ then $\mathscr{F}$ can be the trace on $X$ of zero-sets in $Y$ for many compactifications $Y$ of $X$. For each such $Y, v(\mathscr{F})$ is a subspace of $Y$. More precisely,

THEOREM. If $Y$ is a Hausdorff compactification of $X$ and $\mathscr{F}=\{Z \cap X: Z \in Z[Y]\}$ then $v(\mathscr{F})$ is the intersection of all cozero-sets in $Y$ which contain $X($ Hager $[6,4.2])$. 
Proof. By $2.8, \omega(\mathscr{F}) \geqq Y$; so let $\varphi$ denote the canonical quotient map from $\omega(\mathscr{F})$ onto $Y$. If $S=\bigcap\{Y-Z: Z \in Z[Y]$ and $Z \cap X=\varnothing\}$, then as in the proof of 3.4, $\varphi(v(\mathscr{F})) \subset S$. If $p \in S$ then $\mathscr{U}_{p}=\{Z \cap X: p \in Z \in Z[Y]\} \in v(\mathscr{F})$, and $\varphi\left(\mathscr{U}_{p}\right) \in S$. For any $q \neq p$ there is a zero-set containing $p$ which misses $q$ so $q \notin\left\{(Z \cap X)^{-}\right.$: $p \in Z\}$; thus $\varphi\left(\mathscr{U}_{p}\right)=\{p\}$ and $\varphi(v(\mathscr{F}))=S$. It is not difficult to see that $\varphi$ is one-toone on $v(\mathscr{F})$ and that $\varphi(\omega(\mathscr{F})-v(\mathscr{F}))=Y-S$. Since $\varphi$ is a closed mapping on $\omega(\mathscr{F}), \varphi$ is a homeomorphism on $v(\mathscr{F})$.

3.10 Alo and Shapiro [1] have considered normal, separating intersection rings without requiring nest generation and have used the same procedure to obtain extensions. More precisely, they define $\eta(\mathscr{F})$ to be the family of ultrafilters on $\mathscr{F}$ which have the countable intersection property, where $\mathscr{F}$ is a normal, separating intersection ring. They then ask whether such extensions are realcompact. We will give an example in 3.11 to show that $\eta(\mathscr{F})$ is not always realcompact.

Also, Alo and Shapiro ask whether every realcompactification $Y$ of a space $X$ can be obtained as an $\eta(\mathscr{F})$. If $X$ is a Lindelöf space then $\eta(\mathscr{F}) \subseteq X=v X$, and since a noncompact Lindelöf space has realcompactifications other than $v X$, the answer to their question is negative.

3.11 Let $X$ be the disjoint union of the space $W$, of all ordinals less than the first uncountable ordinal and $D$, the uncountable discrete space of cardinality $c$.

There exists a countable collection $\left\{U_{i}: i \in N\right\}$ of subsets of $D$ such that $U_{i} \supset U_{i+1}$, each $U_{i}$ is uncountable, and $\bigcap\left\{U_{i}: i \in N\right\}=\varnothing$. Define

$$
\mathscr{V}=\left\{V \subset D:\left(V-U_{i}\right) \cup\left(U_{i}-V\right) \text { is countable for some } i \in N\right\} .
$$

(By countable is meant countably infinite, finite or empty.)

Let $\mathscr{F}$ be the family of all zero-sets $Z$ in $X$ of the form either:

(i) $Z \cap W$ contains a tail in $W$ and $Z \cap D$ is a cocountable subset of $D$;

(ii) $Z \cap W$ is compact and $Z \cap D$ is countable; or

(iii) $Z \cap W$ is compact and $Z \cap D \in \mathscr{V}$.

It is a routine task to check that $\mathscr{F}$ is a normal, separating ring, closed under countable intersections.

If $\mathscr{U}$ is an ultrafilter on $\mathscr{F}$ and $\cap \mathscr{U}=\varnothing$, then either $\mathscr{U}$ contains all sets of form (iii) or $\mathscr{U}$ contains a set of form (ii). For if $Z \notin \mathscr{U}$ and $Z$ is of form (iii) then there is a $F \in \mathscr{U}$ such that $F \cap Z=\Phi$. Since $Z \cap D$ is uncountable, $F \cap D$ must be countable so $F$ is of form (ii). Every set of form (ii) is Lindelöf and thus $\mathscr{U}$ fails to have the countable intersection property. On the other hand if all sets of the form (iii) are in $\mathscr{U}$, then $U_{i} \in \mathscr{U}$ for all $i \in N$, and $\cap U_{i}=\varnothing$.

Thus, every ultrafilter on $\mathscr{F}$ with the countable intersection property converges in $X$ but $X$ is not realcompact. Hence $\eta(\mathscr{F})=X$ and is not realcompact.

3.12 Corollary 2.3 shows that the relation between $\mathscr{L}(X)$ and the associated Wallman compactifications $\mathscr{L}^{*}(X)$ is one-to-one. This is not the case between $\mathscr{L}(X)$ and the associated Wallman realcompactifications as the following example shows. 
3.13 Let $X=[0,1]$ with the discrete topology, let $\mathscr{F}_{1}$ be the family of all closed subsets of $X$ with respect to the usual topology on $[0,1]$, and let $\mathscr{F}_{2}$ be the family of all subsets of $X$ which are finite or whose complement is countable.

$\mathscr{F}_{1}$ is a nest-generated intersection ring, $\mathscr{F}_{2} \in \mathscr{L}(X)$, so by Lemma 1.7,

$$
\mathscr{F}=\mathscr{F}_{1}+\mathscr{F}_{2} \in \mathscr{L}(X) .
$$

If $\mathscr{U}$ is an ultrafilter on $\mathscr{F}$ then there is a countable family $\left\{A_{n}\right\}$ in $\mathscr{U}$ such that for each $n \in N, A_{n}=\left[(j-1) / 2^{n}, j / 2^{n}\right]$ for some $j, 1 \leqq j \leqq 2^{n}$, and $A_{n} \supset A_{n+1}$. Now $\bigcap\left\{A_{n}: n \in N\right\}=\{p\}$; so if $\mathscr{U}$ has the countable intersection property, then $\bigcap \mathscr{l}=\{p\}$. Thus $v(\mathscr{F})=X=v X$. However, $\mathscr{F} \neq Z[X]$.

Other examples of this kind have been given by Isbell [9], Henriksen and Johnson [8], and Hager [6], but as certain inverse-closed subalgebras of $C(X)$. We will investigate this relation in the next section.

\section{Inverse-closed subalgebras of $C(X)$.}

4.1 By an algebra on $X$ is meant a subalgebra of $C(X)$ which contains the constants, separates points and closed sets, and is closed under uniform convergence and inversion in $C(X)$. If $A$ is an algebra on $X$, then $A^{*}$ is the set of all bounded functions in $A, H\left(A^{*}\right)$ is the structure space of $A$ (the space of maximal ideals of $A$ with the Stone topology [5]) and $H(A)$ is the intersection of all cozero-sets in $H\left(A^{*}\right)$ which contain $X . H\left(A^{*}\right)$ is a compactification of $X$ and $H(A)$ is a realcompactification of $X$. Clearly, $H\left(C^{*}(X)\right)=\beta X$ and $H(C(X))=v X$.

4.2 Let $A$ be an algebra of $X$. Then $H\left(A^{*}\right)$ is a compactification of $X$ and $A^{*}=C\left(H\left(A^{*}\right)\right)$ [9]. By Lemma 1.4 the trace on $X$ of zero-sets in $H\left(A^{*}\right)$, which is the collection of zero-sets of functions in $A^{*}$, is in $\mathscr{L}(X)$. Again from [9], $A$ is just the family of all continuous functions from $H\left(A^{*}\right)$ to the two-point compactification of the reals, which are realvalued on $X$. Thus if $\mathscr{Z}(A)=\{Z(f): f \in A\}$, then $\mathscr{Z}(A)=\mathscr{Z}\left(A^{*}\right)=\left\{Z \cap X: Z \in Z\left[H\left(A^{*}\right)\right]\right\} \in \mathscr{L}(X)$.

4.3 THEOREM. The mapping $A \rightarrow \mathscr{Z}(A)$ is a one-to-one correspondence between the family of all algebras on $X$ and $\mathscr{L}(X)$.

Proof. Let $\mathscr{F} \in \mathscr{L}(X)$ and let $A_{0}(\omega(\mathscr{F}))=\{f|X| g \mid X: f, g \in C(\omega(\mathscr{F}))$ and $Z(g) \cap X=\varnothing\}$. By Corollary 2.10, if $T$ is a cozero set in $\omega(\mathscr{F})$ containing $X$, then $\beta T=\omega(\mathscr{F})$. Thus, from a proposition in [6], $A_{0}(\omega(\mathscr{F}))$ is an algebra on $X$ and $\omega(\mathscr{F})=H\left(A_{0}^{*}(\omega(\mathscr{F}))\right)$. Thus $\mathscr{F}=\mathscr{Z}\left(A_{0}(\omega(\mathscr{F}))\right)$ and the correspondence is onto. In [9] it is shown that for each algebra $A$ on $X, H\left(A^{*}\right)$ has the property that for each cozero-set $T$ in $H\left(A^{*}\right)$ which contains $X, \beta T=H\left(A^{*}\right)$. Thus by 5.4 of [6], if $A$ is any algebra such that $\mathscr{Z}(A)=\mathscr{F}=\left\{Z \cap X: Z \in Z\left[H\left(A^{*}\right)\right]\right\}$, then $\omega(\mathscr{F})=H\left(A^{*}\right)$. Therefore if $\mathscr{Z}(A)=\mathscr{F}, A=A_{0}(\omega(\mathscr{F}))$ and the correspondence is one-to-one.

4.4 If $A$ is an algebra on $X$ and $\mathscr{Z}(A)=\mathscr{F} \in \mathscr{L}(X)$ then $\omega(\mathscr{F})=H\left(A^{*}\right)$ and $v(\mathscr{F})=H(A)$. This last equality is clear from the definitions of $v(\mathscr{F})$ and $H(A)$.

If $A$ is an algebra on $X$ and $A=C(Y)$ for some space $Y$, then $H(A)=H(C(Y))$ 
$=v Y$ and thus $C(H(A))$ is isomorphic to $C(Y)$ and $A=C(H(A))$. However, as Example 3.13 shows, $A$ need not be $C(Y)$ for any space $Y$.

5. $\mathscr{L}^{*}(X)$.

5.1 The family $\mathscr{K}(X)$ of all Hausdorff compactifications of $X$ is a partially ordered set where $Y_{1} \geqq Y_{2}$ if and only if the identity map on $X$ has a continuous extension from $Y_{1}$ onto $Y_{2} . \beta X$ is the largest member of $\mathscr{K}(X)$ and $\mathscr{K}(X)$ has a smallest member if and only if $X$ is locally compact. If $X$ is locally compact then $\mathscr{K}(X)$ is a complete lattice [3], [11]. It is always the case that any two members of $\mathscr{K}(X)$ have a l.u.b. in $\mathscr{K}(X)$.

Each member of $\mathscr{K}(X)$ is determined by an upper semicontinuous decomposition of $\beta X$. If $\mathscr{D}_{1}$ and $\mathscr{D}_{2}$ determine $Y_{1}$ and $Y_{2}$, respectively, then the decomposition $\mathscr{D}_{3}$ of all intersections of the form $\left\{D_{1} \cap D_{2}: D_{1} \in \mathscr{D}_{1}, D_{2} \in \mathscr{D}_{2}\right\}$ is upper semicontinuous and determines the l.u.b. of $Y_{1}$ and $Y_{2}$, written $Y_{1} \vee Y_{2}$.

The family $\mathscr{L}^{*}(X)$ of compactifications of the form $\omega(X, \mathscr{F})$ for $\mathscr{F} \in \mathscr{L}(X)$ is a subfamily of $\mathscr{K}(X)$ and has the induced partial order. We will show that any two members of $\mathscr{L}^{*}(X)$ have a l.u.b. in $\mathscr{L}^{*}(X)$ and that $\mathscr{L}^{*}(X)$ is a complete lattice whenever $\mathscr{K}(X)$ is.

5.2 THEOREM. If $\mathscr{F}, \mathscr{G} \in \mathscr{L}(X)$ then $\omega(\mathscr{F}) \geqq \omega(\mathscr{G})$ if and only if $\mathscr{G} \subset \mathscr{F}$.

Proof. If $\mathscr{G} \subset \mathscr{F}$ then disjoint sets in $\mathscr{G}$ may be separated by disjoint sets in $\mathscr{F}$ so $\omega(\mathscr{F}) \geqq \omega(\mathscr{G})$ [15]. Conversely, suppose $\omega(\mathscr{F}) \geqq \omega(\mathscr{G})$. If $G \in \mathscr{G}$ then $G=Z \cap X$ for some $Z \in Z[\omega(\mathscr{G})]$. If $\varphi$ is the canonical quotient map from $\omega(\mathscr{F})$ onto $\omega(\mathscr{G})$ then $\varphi^{\leftarrow}[Z] \in Z[\omega(\mathscr{F})]$ so $G=\varphi^{\leftarrow}[Z] \cap X \in \mathscr{F}$.

In fact, whenever $Y_{1}, Y_{2} \in \mathscr{K}(X)$ and $Y_{1} \geqq Y_{2}$, then

$$
\left\{Z \cap X: Z \in Z\left[Y_{2}\right]\right\} \subset\left\{Z \cap X: Z \in Z\left[Y_{1}\right]\right\} \text {. }
$$

5.3 Theorem. If $\mathscr{F}, \mathscr{G} \in \mathscr{L}(X)$ then $\omega(\mathscr{F}+\mathscr{G})$ is the l.u.b. in $\mathscr{L}^{*}(X)$ of $\omega(\mathscr{F})$ and $\omega(\mathscr{G})$.

Proof. $\mathscr{F}+\mathscr{G} \in \mathscr{L}(X)$ and $\mathscr{F}, \mathscr{G} \subset \mathscr{F}+\mathscr{G}$ so by Theorem $5.2 \omega(\mathscr{F}+\mathscr{G}) \geqq \omega(\mathscr{F})$ and $\omega(\mathscr{F}+\mathscr{G}) \geqq \omega(\mathscr{G})$. If $\omega(\mathscr{H}) \geqq \omega(\mathscr{F})$ and $\omega(\mathscr{H}) \geqq \omega(\mathscr{G})$ for some $\mathscr{H} \in \mathscr{L}(X)$, then by Theorem 5.2, $\mathscr{F}$ and $\mathscr{G}$ are contained in $\mathscr{H}$. Thus $\mathscr{F}+\mathscr{G} \subset \mathscr{H}$ and $\omega(\mathscr{H})$ $\geqq \omega(\mathscr{F}+\mathscr{G})$.

5.4 The following lemma shows how the l.u.b. in $\mathscr{L}^{*}(X)$ of $\omega(\mathscr{F})$ and $\omega(\mathscr{G})$ is related to $\omega(\mathscr{F}) \vee \omega(\mathscr{G})$.

LEMMA. If $\mathscr{F}, \mathscr{G} \in \mathscr{L}(X)$ and $Y=\omega(\mathscr{F}) \vee \omega(\mathscr{G})$ then $\{Z \cap X: Z \in Z[Y]\}=\mathscr{F}+\mathscr{G}$.

Proof. If $\mathscr{H}=\{Z \cap X: Z \in Z[Y]\}$ then, by Lemma 1.4, $\mathscr{H} \in \mathscr{L}(X)$. Clearly, $\mathscr{F}$ and $\mathscr{G}$ are contained in $\mathscr{H}$ so $\mathscr{F}+\mathscr{G} \subset \mathscr{H}$. Now $Y=\omega(\mathscr{F}) \vee \omega(\mathscr{G})$ so $\omega(\mathscr{F}+\mathscr{G}) \geqq Y$ by 5.3 and $\mathscr{H} \subset \mathscr{F}+\mathscr{G}$. Thus $\mathscr{H}=\mathscr{F}+\mathscr{G}$.

5.5 We will denote the g.l.b. in $\mathscr{K}(X)$ of $Y_{1}$ and $Y_{2}$, when it exists, by $Y_{1} \wedge Y_{2}$.

LEMMA. If $\mathscr{F} \mathscr{G} \in \mathscr{L}(X)$ and $Y=\omega(\mathscr{F}) \wedge \omega(\mathscr{G})$, then $Y \in \mathscr{L}^{*}(X)$. 
Proof. Let $\mathscr{H}=\{Z \cap X: Z \in Z[Y]\}$. Then $\mathscr{H} \subset \mathscr{F}$ and $\mathscr{H} \subset \mathscr{G}$ so $\mathscr{H} \subset \mathscr{F} \cap \mathscr{G}$. Since $\mathscr{H} \in \mathscr{L}(X)$, by $5.2 \omega(\mathscr{F}) \geqq \omega(\mathscr{H})$ and $\omega(\mathscr{G}) \geqq \omega(\mathscr{H})$. Thus $Y \geqq \omega(\mathscr{H})$. But from 2.8 we know $\omega(\mathscr{H}) \geqq Y$. Thus $\omega(\mathscr{H})=Y \in \mathscr{L}^{*}(X)$.

5.6 THEOREM. If $X$ is locally compact, $\mathscr{L}^{*}(X)$ is a complete lattice.

Proof. If $X$ is locally compact, $\mathscr{K}(X)$ is a complete lattice and by Lemmas 5.4 and $5.5, \mathscr{L}^{*}(X)$ is a lattice. If $\mathscr{F}_{\alpha} \in \mathscr{L}(X)$ for all $\alpha$ in some index set $A$, then $Y=$ $\bigwedge\left\{\omega\left(\mathscr{F}_{\alpha}\right): \alpha \in A\right\} \in \mathscr{K}(X)$ and in precisely the same manner as in Lemma 5.6, $Y \in \mathscr{L}^{*}(X)$. Since $\mathscr{L}^{*}(X)$ has a largest element, $\beta X, \mathscr{L}^{*}(X)$ is a complete lat tice.

5.7 In general, it is known that $\mathscr{K}(X)$ need not be a lattice. This follows from a construction of Isbell [10]: if $X$ is a nonlocally compact, first countable space, then there exists a pair of compactifications of $X$ with no g.l.b. in $\mathscr{K}(X)$. Even in this case, however, $\mathscr{L}^{*}(X)$ may be a lattice; for example, if $X$ is a nonlocally compact, separable metric space, then $X$ is Lindelöf and $\mathscr{L}^{*}(X)=\{\beta X\}$, a trivial lattice. We will use a similar construction to give an example where $\mathscr{L}^{*}(X)$ is not a lattice.

Let $X=N^{*} \times W^{*}-\{(n, \Omega): n \in N\}$ where $N^{*}$ denotes the one-point compactification of the integers and $W^{*}$ denotes the space of all ordinals less than or equal to the first uncountable ordinal $\Omega . \beta X=N^{*} \times W^{*}$ and although $\left(\omega_{0}, \Omega\right),\left\{\omega_{0}\right\}$ $=N^{*}-N$, does not have a countable base, there is a sequence in $\beta X-X$ converging to $\left(\omega_{0}, \Omega\right)$ and this is all that is needed. Let $\mathscr{D}_{1}$ be the decomposition of $\beta X$ whose nonsingleton sets are the pairs $\{(2 n-1, \Omega),(2 n, \Omega)\}$ and $\mathscr{D}_{2}$ the decomposition whose nonsingleton sets are the pairs $\{(2 n, \Omega),(2 n+1, \Omega)\}$. Both $\mathscr{D}_{1}$ and $\mathscr{D}_{2}$ are upper semicontinuous decompositions and thus provide compactifications $Y_{1}$ and $Y_{2}$ in $\mathscr{K}(X)$. Since $\{(n, \Omega): n \in N\}$ is in $\beta X-X$ and converges to $\left(\omega_{0}, \Omega\right) \in X$, there can be no upper semicontinuous decomposition of $\beta X$ which has an element containing $\{(n, \Omega): n \in N\}$. Therefore, $Y_{1}$ and $Y_{2}$ have no g.l.b. in $\mathscr{K}(X)$. Since $X$ is pseudocompact, $\mathscr{K}(X)=\mathscr{L}^{*}(X)$, [6], and $\mathscr{L}^{*}(X)$ is not a lattice.

5.8 Even if $X$ is locally compact, $\mathscr{L}^{*}(X)$ need not be a sublattice of $\mathscr{K}(X)$ as the following example illustrates.

Let $X$ be a discrete space of cardinality $c$. Write $X=A \cup B$ where $A \cap B=\varnothing$ and $A$ and $B$ are countable unions of disjoint sets of cardinality $c, A=\bigcup_{i=1}^{\infty} A_{i}$, $B=\bigcup_{j=1}^{\infty} B_{j}$. Let $\mathscr{F}$ be the intersection ring generated by all subsets $F \subset X$ such that either

(i) $F$ is finite,

(ii) $X-F$ is countable (finite or infinite),

(iii) $F=B_{j}$ for some $j$,

(iv) $F=X-\bigcup\left\{B_{j}: j \in N^{\prime}\right\}$ where $N^{\prime}$ is a finite subset of $N$.

The family $\mathscr{F}_{1}=\{F \subset X: F$ satisfies (i) or (ii) $\} \in \mathscr{L}(X)$. (In fact, $\omega\left(\mathscr{F}_{1}\right)$ is the onepoint compactification of $X$.) The family $\mathscr{F}_{2}=\{F \subset X: F$ satisfies (iii) or (iv) $\}$ is nest generated (for each $F \in \mathscr{F}_{2}, X-F \in \mathscr{F}_{2}$ ) so by Lemmas 1.5 and 1.6 , the intersection ring $\mathscr{F}_{2}^{\prime}$ generated by $\mathscr{F}_{2}$ is also nest generated. Since $\mathscr{F}=\mathscr{F}_{1}+\mathscr{F}_{2}^{\prime}$, by 
Lemma $1.7, \mathscr{F}$ is a nest generated intersection ring and $\mathscr{F}$ is separating because $\mathscr{F}_{1}$ is separating. Thus $\mathscr{F} \in \mathscr{L}(X)$.

Similarly, let $\mathscr{G} \in \mathscr{L}(X)$ be defined as $\mathscr{F}$ with the $B_{j}$ 's replaced by $A_{i}$ 's.

Let $Y=\omega(\mathscr{F}) \vee \omega(\mathscr{G})$. For any set $E \subset X$ we will denote the closure of $E$ in $\omega(\mathscr{F}+\mathscr{G})$ by $\mathrm{cl} E$, in $Y$ by $\operatorname{cl}_{Y} E$, and in $\beta X$ by $E^{-}$. Now, $A \in \mathscr{F}$ and $B \in \mathscr{G}$ so $A, B \in \mathscr{F}+\mathscr{G}$ and thus $\mathrm{cl} A \cap \mathrm{cl} B=\varnothing$. We will show $Y \neq \omega(\mathscr{F}+\mathscr{G})$ by showing $\operatorname{cl}_{Y} A \cap \mathrm{cl}_{Y} B \neq \varnothing$.

Every compactification of $X$ is determined by an upper semicontinuous decomposition of $\beta X$. The decomposition $\mathscr{D}_{1}$ of sets of the form (i) $\{x\}, x \in X$; (ii) $\bar{B}_{j}-B_{j}, j \in N$; or (iii) $(\beta X-X)-\bigcup\left\{\bar{B}_{j}: j \in N\right\}$ is seen to be upper semicontinuous and to give $\omega(\mathscr{F})$. Similarly, the decomposition $\mathscr{D}_{2}$ of sets of the form (i) $\{x\}, x \in X$; (ii) $\bar{A}_{i}-A_{i}, i \in N$; or (iii) $(\beta X-X)-\bigcup\left\{\bar{A}_{i}: i \in N\right\}$ gives $\omega(\mathscr{G})$. It can be seen that the decomposition $\mathscr{D}_{3}$ giving $Y$ is composed of sets of the form

(i) $\{x\}, x \in X$;

(ii) $\bar{A}_{i}-A_{i}, i \in N$;

(iii) $\bar{B}_{j}-B_{j}, j \in N$; or

(iv) $(\beta X-X)-\left[\bigcup\left\{\bar{A}_{i}: i \in N\right\} \cup \cup\left\{\bar{B}_{j}: j \in N\right\}\right]$.

The set $\bar{A}-\bigcup\left\{\bar{A}_{i}: i \in N\right\} \neq \varnothing$ since $\bar{A}_{i} \cap \bar{A}_{j}=\varnothing$ and no countable disjoint union of closed sets is compact. Thus, if $p \in \bar{A}-\bigcup\left\{\bar{A}_{i}: i \in N\right\}$ and $q \in \bar{B}-\bigcup\{\bar{B},: j \in N\}$ then $p$ and $q$ are in the same decomposition element of $\mathscr{D}_{3}$. Therefore if $\varphi$ is the quotient map from $\beta X$ onto $Y, \varphi[\bar{A}]=\mathrm{cl}_{Y} A, \varphi[\bar{B}]=\mathrm{cl}_{Y} B$ and $\varphi(p)=\varphi(q)$; so we see that $\varphi(p) \in \mathrm{cl}_{Y} A \cap \mathrm{cl}_{Y} B$.

$Y-X$ is countable so, by [15], $Y$ is a Wallman compactification of $X$ but $Y \notin \mathscr{L}^{*}(X)$.

5.9 The following example shows that if $\mathscr{F} \mathscr{G} \in \mathscr{L}(X)$ then $\mathscr{F} \cap \mathscr{G}$ need not be in $\mathscr{L}(X)$, even if there is an $\mathscr{H} \in \mathscr{L}(X)$ and $\mathscr{H} \subset \mathscr{F} \cap \mathscr{G}$. Let $X$ be a nonpseudocompact, nonrealcompact space such that $|v X-X| \geqq 2$ and $|\beta X-v X| \geqq 1$. (To see that such spaces exist, let $W^{*}$ be the ordinals less than or equal to the first uncountable ordinal $\Omega$ and let $X=N \times W^{*}-\{(1, \Omega),(2, \Omega)\}$.) Let $p, q \in v X-X$ and $r \in \beta X-v X . p, q$ and $r$ are contained in disjoint zero-set neighborhoods, $Z_{p}, Z_{q}$ and $Z_{r}$. Let $\mathscr{D}_{1}$ be the decomposition of $\beta X$ whose only nonsingleton is $\{p, r\}$ and let $\mathscr{D}_{2}$ be the decomposition whose only nonsingleton set is $\{q, r\} . \mathscr{D}_{1}$ and $\mathscr{D}_{2}$ are upper semicontinuous so the corresponding compactifications $Y_{1}$ and $Y_{2}$ are Hausdorff. Let $\mathscr{F}_{1}=\left\{Z \cap X: Z \in Z\left[Y_{1}\right]\right\}$ and $\mathscr{F}_{2}=\left\{Z \cap X: Z \in Z\left[Y_{2}\right]\right\}$. It is not difficult to show that $Y_{1}=\omega\left(\mathscr{F}_{1}\right)$ and $Y_{2}=\omega\left(\mathscr{F}_{2}\right)$, and that $Z_{p}, Z_{q} \in \mathscr{F}_{1} \cap \mathscr{F}_{2}$. However, the decomposition $\mathscr{D}_{3}$ giving $Y_{3}=Y_{1} \wedge Y_{2}$ has as its only nonsingleton set $\{p, q, r\}$ and since $Z_{p} \cap Z_{q}=\varnothing$, neither is the trace of a zero-set in $Y_{3}$ on $X$.

5.10 From Theorem 3.7 we know that $X$ is Lindelöf or almost compact, if and only if $\mathscr{L}^{*}(X)=\{\beta X\}$. How large can $\mathscr{L}^{*}(X)$ be ? If $X$ is realcompact and not Lindelöf, then from 3.8 we know that $\mathscr{L}^{*}(X)$ is at least countably infinite and if $X$ is not realcompact and not pseudocompact, 3.8 implies that $\mathscr{L}^{*}(X)$ has cardinality at least $2^{c}$. In case $X$ is pseudocompact, Hager [6] has shown that $\mathscr{L}^{*}(X)$ 
$=\mathscr{K}(X)$. Each identification of a pair of points in $\beta X-X$ for $X$ pseudocompact, produces an element of $\mathscr{K}(X)$, hence of $\mathscr{L}^{*}(X)$. For any cardinal $m$ there is a pseudocompact space $X$ such that $|\beta X-X|=m$, [5], and thus the cardinality of $\mathscr{L}^{*}(X) \geqq m$.

\section{BIBLIOGRAPHY}

1. R. A. Alo and H. L. Shapiro, $\mathscr{Z}$-real compactifications and normal bases, J. Austral. Math. Soc. 9 (1969), 489-495.

2. B. Banaschewski, On Wallman's method of compactification, Math. Nachr. 27 (1963), 105-114. MR 28 \#3400.

3. N. Boboc and Gh. Siretchi, Sur la compactification d'un espace topologique, Bull. Math. Soc. Sci. Math. Phys. R. P. Roumaine 5 (53) (1961), 155-165 (1964). MR 32 \#430.

4. O. Frink, Compactifications and semi-normal spaces, Amer. J. Math. 86 (1964), 602-607. MR 29 \#4028.

5. L. Gillman and M. Jerison, Rings of continuous functions, The University Series in Higher Math., Van Nostrand, Princeton, N. J., 1960. MR 22 \#6994.

6. A. W. Hager, On inverse-closed subalgebras of $C(X)$, Proc. London Math. Soc. (3) 19 (1969), 233-257.

7. A. W. Hager and D. G. Johnson, A note on certain subalgebras of $C(X)$, Canad. J. Math. 20 (1968), 389-393. MR 36 \#5697.

8. M. Henriksen and D. G. Johnson, On the structure of a class of archimedean latticeordered algebras, Fund. Math. 50 (1961/62), 73-94. MR 24 \#A3524.

9. J. R. Isbell, Algebras of uniformly continuous functions, Ann. of Math. (2) 68 (1958), 96-125. MR 21 \#2177.

10. ——, Uniform neighborhood retracts, Pacific J. Math. 11 (1961), 609-648. MR 25 \#4487.

11. R. G. Lubben, Concerning the decomposition and amalgamation of points, upper semicontinuous collections, and topological extensions, Trans. Amer. Math. Soc. 49 (1941), 410-466. MR 3, 136.

12. N. A. Sanin, On special extensions of topological spaces, Dokl. Akad. Nauk SSSR 38 (1943), 6-9. MR 5, 45.

13. E. F. Steiner, Normal families and completely regular spaces, Duke Math. J. 33 (1966), 743-745. MR 33 \#7975.

14. - Wallman spaces and compactifications, Fund. Math. 61 (1967/68), 295-304. MR 36 \#5899.

15. A. K. Steiner and E. F. Steiner, Wallman and Z-compactifications, Duke Math. J. 35 (1968), 269-276. MR 37 \#3526.

Iowa State University, AMES, IOWA 50010 